\title{
The Kinematics of Globular Cluster NGC 288
}

\author{
Chan-Kao Chang \\ Institute of Astronomy, National Central University, 32054 Chung-li, \\ Taiwan; rex@phy.ncu.edu.tw
}

Alfred B. Chen, Wean-Shun Tsay, Wen-Ping Chen

Institute of Astronomy, National Central University, 32054 Chung-li, Taiwan

Phillip K. Lu

Department of Astronomy, Yale University, P.O. Box 208101, New Haven, CT 06520, USA

\begin{abstract}
The mean radial velocity of NGC 288 (accuracy $5.5 \mathrm{~km} / \mathrm{s}$ ) is determined to be $-56.3 \pm 20.1 \mathrm{~km} / \mathrm{s}$ which, when combined with the mean proper motion (Guo, 1995), yields a peculiar velocity with respect to the LSR of $(u, v, w)=(29.7 \pm 18.1,-258.6 \pm 18.3,62.3 \pm 20.3) \mathrm{km} / \mathrm{s}$. This implies that NGC 288 moves in a retrograde sense with the Galactic rotation. We also derived the effective temperatures for stars in our sample and, as a corroborative effort, compared with those estimated previously from the BATC data (Tsai 1998) by spectral energy distribution fitting. We demonstrate that the BATC/SED fitting is an appropriate and efficient way to estimate the effective temperature of a star.
\end{abstract}

\section{Introduction}

The globular cluster NGC 288 has an obvious blue horizontal branch with intermediate metallicity (the second parameter problem). Its location near the South Galactic Pole makes it a useful probe to study the gravitational force and the local mass density of the Milky Way. In the past two decades, however, investigation on the metal abundances and the age of NGC 288 has been mainly by photometry. Kinematics information has been scarce. To remedy this, spectra of NGC 288 were obtained, and radial velocities of known member stars were measured. In addition, stellar effective temperature was derived for each star.

The WIYN/Hydra Multi-Fiber positioner was used in 1988 to obtain the spectra for radial velocity determination and spectral classification. Typical integration times were between 1000 and 4000 seconds, with a spectral resolution of $2.2 \AA$. The internal accuracy of our data, as determined by cross analysis of about 100 solar spectra in a single sky flat exposure, is about $5.5 \mathrm{~km} / \mathrm{s}$. 


\section{Results}

The mean radial velocity of NGC 288 , as determined from 23 member stars, is $-56.3 \pm 20.1 \mathrm{~km} / \mathrm{s}$ which, when combined with the proper motion previously determined by Guo (1995), yields a space velocity with respect to the solar system $(U, V, W)=(38.7 \pm 18.1,-270.6 \pm 18.3,55.3 \pm 20.3) \mathrm{km} / \mathrm{s}$. Adopting a peculiar velocity for the sun $(u, v, w)=(-9,12,7) \mathrm{km} / \mathrm{s}$ (Mihalas, 1981), we obtained the peculiar velocity for NGC 288 to be $(u, v, w)=(29.7 \pm 18.1,-258.6 \pm$ $18.3,62.3 \pm 20.3) \mathrm{km} / \mathrm{s}$. The total velocity of NGC 288 with respect to the LSR is thus $267.7 \pm 32.8 \mathrm{~km} / \mathrm{s}$ toward $l=261.3^{\circ}, b=13.5^{\circ}$. If the rotational velocity of the LSR with respect to the Galactic center is $-220 \mathrm{~km} / \mathrm{s}$ (Mihalas, 1981), NGC 288 would be rotating about the Galactic center with $-38 \pm 18.3 \mathrm{~km} / \mathrm{s}$, that is, in a retrograde sense.

We also derived the effective temperatures, $T_{\text {eff }}$, of each star from its spectral type and, as a corroborative effort, compared with that derived from the BATC data by the SED fitting technique. The agreement is in general reasonable (Fig.1), suggesting that the intermediate-band multi-color fitting technique is appropriate in temperature determination, with higher efficiency than spectral observations.

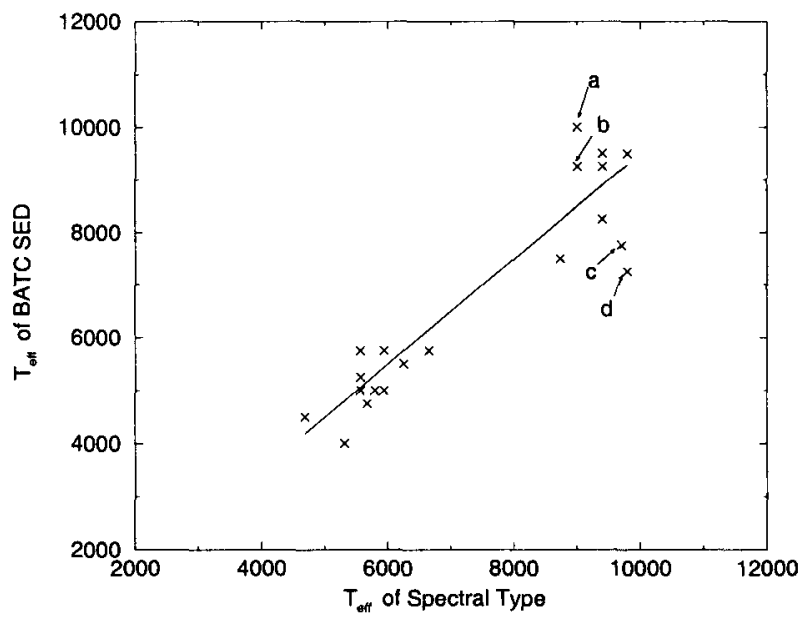

Figure 1. Comparison between the effective temperature determined from spectral classification ( $\mathrm{x}$-axis) and from BATC/SED fitting ( $\mathrm{y}-$ axis). The agreement is reasonable, except at high temperatures for which the BATC data seem less sensitive to.

\section{References}

Chen, A.B.-C., Tsay, W.-S., Tsai, W.-S., Lu, P. K. 2000, AJ, 120, 2569

Guo, X. 1995, Ph.D dissertation, Yale University

Mihalas, D., Binney, J. 1981, Galactic Astronomy: Structrue and Kinematics, 2nd ed., (Freeman) 